Images dans le monde ibérique et ibéricoaméricain

$6 \mid 2013$

Images dans le monde ibérique et ibéro-américain

\title{
L'émergence du contrôle de constitutionnalité en Bolivie
}

De l'indépendance à la constitution bolivienne de 2009

Jean-René Garcia

\section{(2) OpenEdition}

Journals

Édition électronique

URL : http://journals.openedition.org/agedor/781

DOI : 10.4000/agedor.781

ISSN : 2104-3353

Éditeur

Laboratoire LISAA

Référence électronique

Jean-René Garcia, "L'émergence du contrôle de constitutionnalité en Bolivie », L'Âge d'or [En ligne],

6 | 2013, mis en ligne le 01 mars 2013, consulté le 19 avril 2019. URL : http://journals.openedition.org/ agedor/781; DOI : 10.4000/agedor.781 
Jean-René GARCIA

Université Paris 13 - PRES Sorbonne Paris Cité, UFR Droit, Sciences Politiques et Sociales, Laboratoire CERAL (Centre de Recherche sur l'Action Locale)

\section{L'ÉMERGENCE DU CONTRÔLE DE CONSTITUTIONNALITE EN BOLIVIE DE L'INDEPENDANCE À LA CONSTITUTION BOLIVIENNE DE 2009}

Résumé : Avec la Constitution de 2009, le pouvoir exécutif bénéficiait d'une marge de manœuvre importante pour l'élaboration des politiques publiques et, à ce titre, il demeurait un pouvoir exécutif "fort". Cependant, il devait désormais inscrire son action dans le cadre strictement légal de la Constitution sous peine de voir ces mesures remises en cause par le Tribunal Constitutionnel Plurinational. En fait, les débats constitutionnels lors de la Constituante de 2007 à 2008 ne portaient pas tant sur la remise en cause d'une juridiction constitutionnelle qui fonctionnait déjà depuis la réforme de 1994 que sur l'élaboration d'une Constitution qui permettrait une meilleure participation des Indiens au système politique du pays.

Mots-clés : Contrôle de constitutionnalité - Bolivie - Constitution de 2009 - Jurisprudence Droit - Cour suprême - Tribunal

Resumen: Con la Constitución de 2009, el Poder Ejecutivo se beneficiaba de un margen de maniobra importante para la elaboración de políticas públicas, en ese sentido, permanecía como un Poder Ejecutivo «fuerte». Sin embargo, en adelante, debía inscribir su acción dentro del marco estrictamente legal de la Constitución bajo pena de ver sus medidas cuestionadas por el Tribunal Constitucional Plurinacional. En efecto, los debates constitucionales durante la Constituyente de 2007 a 2008 no trataron tanto sobre el cuestionamiento de una jurisdicción constitucional, que ya funcionaba desde la reforma de 1994, sino sobre la elaboración de una Constitución que permitiría una mayor participación indígena en el sistema político del país.

Palabras clave : Control de constitucionalidad - Bolivia - Constitución de 2009 - Jurisprudencia Derecho - Corte Suprema - Tribunal 


\section{INTRODUCTION}

Afin d'analyser l'émergence d'un contrôle de constitutionnalité en Bolivie, il convient de revenir sur les débats concernant la notion de droit en Amérique latine. Ceux-ci se sont centrés autour des contradictions philosophiques entre un droit naturel, le « jusnaturalisme » dont Jean Bodin était le précurseur ${ }^{1}$, et un droit dépendant de l'évolution de la société, le "positivisme» théorisé par Auguste Comte. Pour les défenseurs du «jusnaturalisme», il existait une certaine universalité des valeurs qui se situait au-delà des cultures de toute société. À l'inverse, pour le "positivisme», le droit dépendait essentiellement d'un processus rationnel de compréhension de la société indépendamment de la culture. Pendant longtemps, le «positivisme » fut la théorie dominante en Amérique latine. Il s'agissait pour les juristes latino-américains, ardents lecteurs d'Auguste Comte, de créer des systèmes juridiques nationaux imprégnés des théories «positivistes $»^{2}$. Mais dans les débats juridiques relatifs au «jusnaturalisme» et au "positivisme», les effets de la «culture du droit » en tant que telle ne furent pas pris en compte. Pourtant, il existerait bien selon nous une relation entre la «culture du droit » et le «capital social » de ces sociétés qui dépasserait le seul débat entre « jusnaturalisme » et « positivisme ».

Or, cette relation a pu entraîner une certaine contradiction. En effet, d'une part, l'imprégnation du droit dans les sociétés latino-américaines a pu agir comme un facteur d'organisation des rapports sociaux car la «culture juridique » faisait alors partie intégrante de cette société. Mais d'autre part, la prolifération d'un nombre important d'avocats, et par conséquent de procès, pouvait être aussi analysée comme un facteur de querelles entre les citoyens attentatoires des rapports sociaux.

Ce paradoxe est symptomatique des difficultés d'analyse du rôle du droit dans les sociétés latino-américaines. Car, au niveau de l'organisation sociale, le droit avait pour objectif d'émettre les prescriptions normatives destinées à réguler le comportement des citoyens. Mais le droit avait aussi en Amérique latine une fonction philosophique qui résidait dans l'idée de fondation d'une société. Cette fonction fondatrice se retrouvait bien évidemment aux États-Unis où la Constitution de 1787, issue des pères fondateurs presque déifiés, était perçue comme un texte sacré. En Bolivie, la fonction fondatrice du droit acquit une légitimité historique fondamentale grâce au « législateur originel » Simón Bolívar. Le Libérateur devint celui qui, par le texte constitutionnel, fonda une nouvelle société avec des mécanismes qu'il estimait en adéquation avec les sociétés des pays d'Amérique latine qu'il avait libérés. À l'inverse des cultures juridiques, par exemple anglo-saxonnes, qui plaçaient le juge au centre des mécanismes assurant le respect du droit, garant de l'ordre politique, les juristes latino-américains se référaient aux «grands législateurs » comme Bolívar, qui auraient conçu un système constitutionnel parfait, dans lequel le juge régulateur des conflits n'avait pas une place prépondérante.

La Bolivie constitua un des exemples les plus significatifs de ce phénomène. Historiquement, la Bolivie, depuis sa création en 1825, appartenait à la culture romanogermanique essentiellement de droit écrit. On y observa donc une certaine sacralisation de l'écrit que l'on retrouvait notamment dans la volonté de parfaire les textes constitutionnels et dans la rédaction, peu après l'indépendance, de codes civils inspirés du code napoléonien. Cette tradition juridique attribuait théoriquement un rôle essentiel aux textes

\footnotetext{
1 Jean Bodin, Les Six Livres de la République, Paris, LGF, 1997.

2 Sur ce sujet, on pourra consulter Ramiro Villaroel Claure, Sociología, La Paz, Bolivia, Libreria Editorial "Juventud", 1999, pp. 40-50.
} 
écrits et au législateur. Elle s'opposait à la méthode inductive anglo-saxonne qui consistait à consacrer comme solution à des cas juridiques des précédents judiciaires qui obtenaient ainsi la valeur de règle applicable au cas concret. Mais le système juridique bolivien évolua vite vers une sorte «d'hybridation » avec le modèle européen faisant référence au texte écrit d'une Constitution coexistant avec un système juridictionnel inspiré de la common law.

«L'hybridation » du système juridique bolivien va suivre une dialectique historique constitutionnelle. En effet, c'est sous le gouvernement de Santa Cruz, en 1834, qu'est adopté le code civil bolivien inspiré du code napoléonien français ${ }^{3}$. Ce code civil coexista avec un système constitutionnel doté d'un pouvoir exécutif fort. "L'hybridation » du système politique bolivien se renforça avec l'élargissement, dans la Constitution de 1851, du rôle de la Cour Suprême déclarée compétente en matière de contrôle de constitutionnalité.

La seconde étape de ce processus «d'hybridation» se situa lors de l'adoption de la Constitution bolivienne de 1880. Cette Constitution consacra le consensus sur le pouvoir exécutif ambivalent républicain parallèlement à un processus de révision des codes civils. Mais l'évolution de cette «hybridation » du système politique bolivien se fit jour surtout lors du processus de consolidation démocratique des années 1990. En effet, la réforme constitutionnelle de 1994 allait en fait permettre une constitutionnalisation de l'ordonnancement juridictionnel hybride de la Bolivie notamment avec la création d'un Tribunal Constitutionnel ${ }^{4}$. Cette création d'un Tribunal Constitutionnel démontrait la volonté de la classe politique de renforcer l'application effective des droits classiques présents dans le texte constitutionnel, mais aussi de reconnaitre de nombreux nouveaux droits, en particulier les droits indigènes.

Il y eut aussi, avec cette réforme constitutionnelle, un processus de réorganisation juridictionnelle. Il s'agissait de renforcer la séparation des pouvoirs à l'intérieur du pouvoir judiciaire lui-même. Pour atteindre cet objectif, la réforme constitutionnelle de 1994 puis la Constitution de 2009 créèrent plusieurs entités judiciaires telles que le Conseil de la Judicature, le Défenseur du Peuple et le Tribunal Constitutionnel. Or, au lieu de se compléter, ces entités judicaires eurent davantage pour effet de se concurrencer. En effet, ce renforcement de «l'hybridation» du système institutionnel bolivien juxtaposait sur un même plan des institutions issues aussi bien de la pratique européenne que de celle des États-Unis, mais aussi de la reconnaissance de droit coutumier pour les indigènes.

Cette multiplication des entités judiciaires amena donc une concurrence des compétences entre ces différentes institutions. Ce processus «d'hybridation » donna lieu à une évolution des notions classiques du droit en Bolivie. Mais c'est surtout la Constitution de 2009 qui fit véritablement bouger les frontières du droit et renforça le processus « d'hybridation ». L'article 179 de la Constitution de 2009 énonçait les organes d'un nouvel ordonnancement juridique du pays. On trouvait le Tribunal Suprême de Justice, les Tribunaux départementaux, les Tribunaux de sentence, les Tribunaux et juges "agroenvironnementaux », la juridiction indigène originaire paysanne exercée par ses propres autorités ainsi que des juridictions spécialisées déterminées par la loi ${ }^{5}$. Par ailleurs, l’alinéa 2 de l'article 179 de la Constitution de 2009 précisait explicitement que la juridiction ordinaire

\footnotetext{
${ }^{3}$ República de Bolivia, Redactor de la Convención Nacional del año 1834, La Paz, Bolivia, Edición República de Bolivia, Litografías e Imprentas Unidas, 1926,., p. 56.

4 María Verónica Oblitas F., Ley del Tribunal Constitucional, ley No1836 del $1^{\circ}$ de Abril 1988, La Paz, Cochabamba, Bolivia, Comentada y Concordada, Colección Jurídica "Guntentag", Editorial Los Amigos del Libro, 1998.

5 “Artículo 179 de la Constitución Política del Estado promulgada el 7 de febrero de 2009”, República de Bolivia, Constitución Política del Estado promulgada el 7 de febrero de 2009, La Paz, Bolivia, Colinas de Santa Rita Alto Auquisamana, agosto 2009., p. 71.
} 
avait la même valeur et se trouvait au même niveau hiérarchique que la juridiction indigène originaire paysanne ${ }^{6}$. La justice constitutionnelle était toujours exercée par le Tribunal Constitutionnel Plurinational et le Défenseur du peuple subsistait aussi dans le nouveau texte constitutionnel.

Il s'agira donc dans cet article d'analyser l'émergence du contrôle de constitutionnalité en Bolivie depuis l'indépendance jusqu’à la Constitution de 2009. Pour ce faire nous verrons dans un premier temps la genèse du contrôle de constitutionnalité en Bolivie (I) puis, par la suite, nous étudierons la consécration de contrôle de constitutionnalité dans la Constitution bolivienne de 2009 (II).

\section{GENÈSE DU CONTRÔLE DE CONSTITUTIONNALITÉ EN BOLIVIE}

Historiquement, le contrôle de constitutionnalité n'existait pas dans les premières Constitutions boliviennes. Il existait bien une Cour Suprême dans les Constitutions de $1826^{7}, 1831^{8}, 1834^{9}, 1839^{10}$ et $1843^{11}$. Mais cette Cour Suprême constituait avant tout une juridiction de troisième degré, statuant en cassation sur les affaires qui avaient fait l'objet d'un appel, d'une sentence rendue par une juridiction de second degré dénommée Cour de District $^{12}$. L'article 110 de la première Constitution bolivienne de 1826, qui définissait les attributions de la Cour Suprême, sera d'ailleurs repris, pour l'essentiel, dans les Constitutions postérieures, jusqu'en $1843^{13}$. Mais le contrôle de constitutionnalité des lois n'était pas inscrit dans ces Constitutions. Il fallut donc attendre 1851 pour qu'apparaisse pour la première fois la notion de contrôle de constitutionnalité en Bolivie. En fait, le

\footnotetext{
6 "Artículo 179 inc 2 de la Constitución Política del Estado promulgada el 7 de febrero de 2009”, ibidem, p. 71. 7 “Artículo 110 de la Constitución Política de 1826", Ramón Salinas Mariaca, Las Constituciones de Bolivia, La Paz, Bolivia, Talleres-Escuela de Artes Gráficas del Colegio Don Bosco, 1989, pp. 32-33.

8 “Artículo 113 de la Constitución Política de 1831", ibidem, p. 53.

9 “Artículo 115 de la Constitución Política de 1834”, ibidem, p. 75.

10 “Artículo 93 de la Constitución Política de 1839”, ibidem, p. 97.

11 “Artículos 72, 73 de la Constitución Política de 1843", ibidem, p. 117.

12 "Artículo 110 inc 9 de la Constitución Política del Estado de 1826", ibidem, pp. 32-33.

${ }^{13}$ L'article 110 de la Constitution de 1826 précisait : Les attributions de la Cour Suprême de Justice sont les suivantes : 1 - Connaître des actions en justice pour causes criminelles du Vice-Président de la République, des Ministres d'État et des membres des Chambres quand le Corps Législatif décréterait qu'il y a lieu d'intenter une action en justice.

2 - Connaître de toutes les actions contentieuses en justice concernant le patronato national.

3 - Examiner les bulles, brèves et rescrits quand ils sont en relation avec des matières civiles.

4 - Connaître des actions contentieuses en justice concernant les ambassadeurs, ministres résidents, consuls et agents diplomatiques.

5 - Connaître des actions de révocation en justice des magistrats des Cours de District Judiciaire et préfets départementaux.

6 - Résoudre les conflits de compétence des Cours de District entre elles et les conflits de compétence entre les Cours et les autres autorités.

7 - Connaître en troisième instance la résidence de tout employé public.

8 - Entendre les doutes des autres tribunaux sur la comprébension de certaines lois, et consulter l'Exécutif pour qu'il promeuve la déclaration appropriée dans les Chambres.

9 - Connaître des recours en nullité qui seraient intentés contre les sentences émises en dernière instance par les Cours de District.

10 - Examiner l'etat d'avancement des actions civiles et criminelles en justice en attente devant les Cours de District, par les moyens que la loi établit.

11 - Exercer en dernier lieu la baute faculté de direction, économique et correctionnelle, sur les tribunaux et les juges de la Nation. "Artículo 110 de la Constitución Política del Estado de 1826”, ibidem, p. 32-33.
} 
contrôle de constitutionnalité fut évoqué à l'occasion de débats de l'Assemblée Constituante de 1851. Les constituants boliviens de 1851 s'inspirèrent essentiellement du rôle de la Cour Suprême nord-américaine. À propos de l'influence de la Cour Suprême des États-Unis sur l'émergence du contrôle de constitutionnalité dans la Constitution bolivienne de 1851, Hugo Galindo Decker précisait : « Nous pouvons constater sans aucun doute l'influence du modèle nord-américain parce que le contrôle de constitutionnalité qu'exerçait la Cour Suprême dans les procédures d'inconstitutionnalité était issu de la Cour Suprême nord-américaine $»^{14}$.

Dès lors, la Constitution de 1851 tenta d'instaurer une disposition qui permettait à la Cour Suprême de bénéficier de prérogatives similaires à celles de la Cour Suprême des États-Unis. L'article 82 de la Constitution 1851 disposait ainsi que : «Le Pouvoir Judiciaire est exercé par la Cour Suprême, les (Cours) Supérieures et juges de la République. Il leur appartient en priorité le pouvoir de juger et d'appliquer cette Constitution de préférence aux lois, et les lois de préférence à d'autres résolutions $»^{15}$.

Ce contrôle, qualifié de «diffus» par la doctrine juridique bolivienne ${ }^{16}$, était exercé théoriquement par la Cour Suprême à l'occasion de procès traités en dernière instance, ou des Cours de District (juridictions de second degré) qui examineraient, à l'occasion d'un procès en cours, la constitutionnalité de la norme incriminée. Il faut préciser que, selon la doctrine bolivienne, dès 1851, le contrôle de constitutionnalité pouvait porter sur les lois votées par le législatif, mais aussi sur les décrets pris par le pouvoir exécutif, et sur toutes autres normes émises par un fonctionnaire public ou une autorité administrative ${ }^{17}$. Dans ce type de contrôle, la Cour Suprême pouvait prendre une "résolution d'inconstitutionnalité » lorsqu'une norme émise par une autorité publique n'était pas conforme à la Constitution, aux lois et autres normes. Cette "résolution d'inconstitutionnalité » ne pouvait s'appliquer qu'entre les parties d'un procès. La Cour Suprême devait déclarer alors l'inapplicabilité de la norme en l'espèce, et l'écarter dans le procès en cours. Mais elle ne pouvait l'annuler totalement. Avec la Constitution de 1861, la Cour Suprême vit théoriquement renforcer ses attributions en matière de contrôle de constitutionnalité ${ }^{18}$. L'article 65 de la Constitution de 1861 était d'ailleurs plus élaboré dans sa rédaction sur le rôle de la Cour Suprême dans ce domaine et précisait : «La Cour Suprême pourra connaitre des affaires de droit pur dont la décision dépend de la constitutionnalité ou de l'inconstitutionnalité des lois ${ }^{19}$.

Il n'existait pas encore, dans la Constitution de 1861, de saisine par les citoyens des tribunaux de première instance ou de la Cour Suprême pour étudier la constitutionnalité d'une norme. Mais les juridictions pouvaient théoriquement s'autosaisir, et si elles le désiraient, examiner le contrôle de constitutionnalité d'une loi à l'occasion d'un procès. La Cour Suprême examinait ensuite la constitutionnalité des décisions prises par les juridictions de première instance. Les Constitutions boliviennes postérieures continuèrent à

\footnotetext{
${ }^{14}$ Hugo Galindo Decker, Tribunal Constitucional, La Paz, Bolivia, Editorial Jurídica “Zegada”, República de Bolivia, 1994, p. 26.

15 “Artículo 82 de la Constitución Política de de 1851”, Ramón Salinas Mariaca, Las Constituciones de Bolivia, op. cit., p. 133.

${ }^{16}$ Hugo Galindo Decker, Tribunal Constitucional, op. cit., p. 17.

${ }^{17}$ Lorsque nous examinons le contrôle de constitutionnalité en Bolivie, nous reprenons le terme de "norme" qui désigne, dès les premières Constitutions, l'ensemble des actes juridiques soumis à ce contrôle : lois votées par le pouvoir législatif, décrets du pouvoir exécutif, et tout type d'acte juridique émis par un fonctionnaire représentant l'État.

18 Carlos Castro Rodríguez, Historia Judicial de Bolivia, op. cit., p. 208.

19 “Artículo 65 inc 2 Constitución Política de 1861", Ramón Mariaca Salinas, Las Constituciones de Bolivia, op. cit., p. 148.
} 
reconnaittre à la Cour Suprême le contrôle de constitutionnalité de manière similaire ${ }^{20}$. Ce fut la Constitution de 1878 qui instaura un « recours direct en nullité » contre les normes inconstitutionnelles accessibles aux citoyens ${ }^{21}$. En effet, selon l'article 111 de la Constitution de 1878, la Cour Suprême était compétente pour :

« $1^{\circ}$ Connaitre des recours en nullité conformément aux lois et statuer dans le même temps sur la question principale. $2^{\circ}$ Connaittre en unique instance des sujets de droit pur dont la décision dépend de la constitutionnalité ou de l'inconstitutionnalité des lois, décrets et tout type de résolution $»^{22}$.

En fait, cet article octroyait la possibilité aux citoyens boliviens de saisir la Cour Suprême en intentant un « recours direct en nullité » au motif d'«absence de juridiction » contre tout type de norme émise par un fonctionnaire public qui ne disposait pas de ce pouvoir selon la Constitution, lois, décrets ou toutes autres normes émises par une autorité publique. Dans ce cas, la Cour Suprême avait la possibilité de déclarer une norme nulle si elle était émise par un fonctionnaire qui n'avait pas de compétence juridique en la matière. La Cour Suprême exerçait ainsi par le biais de ce recours un contrôle de constitutionnalité sur la norme incriminée. La haute juridiction qui pouvait être saisie en vertu du « recours direct en nullité » devait constater si l'autorité publique qui avait émis cette norme bénéficiait, selon la Constitution, de la compétence juridique pour le faire. Ce recours était applicable uniquement lorsque l'on observait ce que le droit bolivien dénommait une "absence de juridiction », c'est-à-dire lorsqu'il n'y avait pas de base légale à l'origine de la norme émise par l'autorité publique ou lorsque la Constitution n'attribuait pas directement ce pouvoir à un fonctionnaire qui en avait fait usage. Il s'agissait en fait, avec cette procédure, d'instaurer un moyen de contrôler la séparation des pouvoirs en sanctionnant celui qui enfreignait les attributions d'un autre pouvoir et donc portait atteinte à la hiérarchie des normes imposée par la Constitution. Il faut noter que le contrôle de constitutionnalité devait, selon cet article, porter sur les normes qui ne respectaient pas la Constitution mais aussi les lois, décrets et autres normes émis par une autorité publique.

Mais le contrôle de constitutionnalité instauré par la Charte de 1851, confirmé par les autres Constitutions et notamment celles de 1878 et de 1880, était avant tout symbolique. Il ne fut appliqué que très rarement au cours de l'histoire bolivienne. De 1851 à 1878, la gazette judiciaire bolivienne ne fait état d'aucun arrêt ayant eu à traiter un cas de recours pour inconstitutionnalité d'une norme ${ }^{23}$. De 1878 à 1893, on trouvait seulement un cas de jurisprudence fondé sur le « recours direct en nullité ». Il s'agissait, en l'espèce, d'un arrêt de la Cour Suprême statuant sur la plainte d'un particulier contre un juge de première instance qui prétendait le faire payer pour les frais de procédure relatifs à un procès.

Le juge de première instance se fondait sur un décret du pouvoir exécutif qui avait institué des droits de paiement pour les frais de justice. La Cour Suprême, saisie par le particulier sur la base d'un «recours en nullité » d'une norme pour inconstitutionnalité,

\footnotetext{
${ }^{20}$ Le contrôle de constitutionnalité exercé par la Cour Suprême est reconnu dans l'article 79, inc 2 de la Constitution de 1868, l'article 82 inc 2 de la Constitution de 1871, l'article 111 inc 2 de la Constitution de 1878, l'article 111 inc 2 de la Constitution de 1880, l'article 143 inc 5 de la Constitution de 1938, Ramón Salinas Mariaca, Las Constituciones de Bolivia, op. cit.

${ }^{21}$ Francisco Fernández Segado, "La Jurisdicción constitucional en la reforma de la constitución de Bolivia de 1994”, Comisión andina de juristas, Una mirada a los Tribunales Constitucionales, las experiencias recientes, Lima, Perú, Edición Konrad Adenauer Stiffung, Septiembre 1995, p. 69.

22 “Artículo 111 de la Constitución Política de 1878”, Ramón Salinas Mariaca, Las Constituciones de Bolivia, op. cit., p. 203.

${ }^{23}$ Jaime Urcullo Reyes, "El control constitucional en Bolivia antes de 1994", Comisión andina de juristas, Una mirada a los Tribunales Constitucionales, las experiencias recientes, Lima, Perú, Edición Konrad Adenauer Stiffung, Septiembre 1995, p. 56.
} 
donna raison au demandeur et déclara que «l'administration de la justice était gratuite et que la faculté d'émettre des impôts était du domaine du pouvoir législatif »"

Cet arrêt fut le premier exemple de contrôle de constitutionnalité sur la base d'un « recours en nullité » d'une norme émanant d'un décret émis par le pouvoir exécutif. Par la suite, le contrôle de constitutionnalité ne fut que très peu appliqué dans la seconde moitié du XIX ${ }^{\mathrm{e}}$ siècle. En fait, il existait deux raisons à cette situation.

En premier lieu, il existait une méconnaissance totale de la part des juges et avocats des procédures juridiques nécessaires à la déclaration d'inconstitutionnalité d'une norme ${ }^{25}$. De plus, le manque de formation des juges, le délai trop long des décisions, et la soumission de l'organe judiciaire à l'exécutif, notamment sous les périodes de dictature, rendaient inefficaces les recours destinés à déclarer l'inconstitutionnalité d'une norme émise par les pouvoirs exécutif ou législatif ${ }^{26}$.

En second lieu, la doctrine juridique qui inspira les juges de la Cour Suprême à partir de 1851 estimait que le contrôle de constitutionnalité d'une loi adoptée par le Parlement ou d'un autre acte du pouvoir exécutif constituait une atteinte au principe de séparation des pouvoirs $^{27}$. Dès lors, tout au long du $\mathrm{XIX}^{\mathrm{e}}$ siècle et malgré l'inscription théorique dans le texte des Constitutions de la possibilité de contrôler la constitutionnalité des lois et des décrets, la juridiction suprême ne s'estimait pas compétente pour exercer ce contrôle. La Cour Suprême estimait qu'en exerçant un contrôle de constitutionnalité elle se serait substituée ainsi aux pouvoirs exécutif et législatif ${ }^{28}$. La Cour Suprême déclarait à ce propos « qu'elle ne pouvait porter atteinte au principe de la séparation des pouvoirs, base de l'organisation politique $»^{29}$.

En troisième lieu, la Cour Suprême estimait qu'elle participait déjà elle-même à l'élaboration de la loi et parfois même à la «rédaction complète de codes » par des consultations préalables demandées par les pouvoirs exécutif et législatif avant la promulgation des lois. Dès lors, la Cour Suprême se refusait à toute intervention postérieure dans le contrôle de constitutionnalité de la loi. Cette doctrine fut maintenue dans l'histoire bolivienne par l'ensemble des présidents des Cours Suprêmes boliviennes. Dans un rapport adressé au Sénat en 1884, les membres de la Cour Suprême, Dalence, Sanjinés, Calvo, Carrilo, Terrazas y Boeto, précisèrent :

«Le Pouvoir Judiciaire applique la loi dans les questions concrètes qui se présentent à lui, il ne peut ni l'abroger ni la modifier [...]. Si l'on admettait des demandes directes pour inconstitutionnalité, on conférerait à la Cour Suprême la possibilité de réviser et corriger les actes des autres pouvoirs et, dès lors, on intervertirait les principes constitutionnels [...]. La Cour Suprême peut seulement statuer quand il y a un litige entre particuliers. Dans ce cas, elle écarte l'application de la loi pour le cas d'espèce mais elle ne peut abroger la loi. De cette manière, la Cour Suprême n'exerce pas de fonctions législatives mais des fonctions judiciaires; dans la pratique, elle laisse inefficace la disposition qui ne tardera pas à être retirée par celui qui l’a émise $»^{30}$.

\footnotetext{
${ }^{24}$ Corte Suprema de Justicia, Gaceta Judicial Boliviana, La Paz, Bolivia, Ediciones de la Corte Suprema de Justicia, 1893, n 478, pp. 10-14.

${ }^{25}$ Nestor Pedro Sagüés, Recursos Extraordinarios, Buenos Aires, Argentina, Ed. Astrea, 1989, p. 3.

${ }^{26}$ José Antonio Rivera S., Reformas constitucionales, avances, debilidades y temas pendientes, La Paz, Bolivia, Ediciones Kipus, 1999, p. 143.

${ }_{27}$ Corte Suprema, Discursos de los presidentes de la Corte Suprema, Sucre, Bolivia, Editorial República de Bolivia, 1927, p. 124.

${ }^{28}$ Ibidem, p. 121.

${ }^{29}$ Hugo Galindo Decker, Tribunal Constitucional, op. cit., p. 29.

${ }^{30}$ Luis Paz, La Corte Suprema de Justicia en Bolivia, Sucre, Bolivia, Imprenta Bolívar, 1910, p. 178.
} 
Ce rapport, qui fut réellement le premier document publié par la doctrine juridique bolivienne depuis la création du contrôle de constitutionnalité dans la Charte de 1851, précisait par ailleurs la procédure des «recours en nullité » pour inconstitutionnalité. Le demandeur, lorsqu'il estimait à l'occasion d'un litige avoir été lésé par une norme inconstitutionnelle, devait s'adresser directement à la Cour Suprême, mais il ne pouvait diriger sa demande ni contre le pouvoir législatif, ni contre le pouvoir exécutif, ni contre le pouvoir judiciaire, mais contre le fonctionnaire public intuitu personae qui avait émis une norme considérée comme inconstitutionnelle ${ }^{31}$. La Cour Suprême rendait ensuite une "déclaration» reconnaissant ou infirmant la constitutionnalité de la norme incriminée. Ainsi, de 1851 à 1903, on ne trouvait, excepté le "recours en nullité » de 1893 cité précédemment, aucun recours pour inconstitutionnalité dans la jurisprudence de la Cour Suprême de Justice. Au début du XX $\mathrm{XX}^{\mathrm{e}}$ siècle, en 1903, confirmant cette doctrine, le président de la Cour Suprême José Maria Vada estimait qu'on ne pouvait étudier la constitutionnalité d'une loi dans l'abstrait sans la mettre en relation avec le pouvoir dont elle émanait et que dès lors la Cour Suprême ne pouvait s'ériger en pouvoir censeur direct des autres pouvoirs.

Luis Paz, président de la Cour Suprême en 1921, confirmait encore cette doctrine en précisant que :

«La Cour Suprême, en s'inspirant de la pratique de la Cour Fédérale de la grande République $^{32}$, a seulement compétence pour étudier la constitutionnalité d'une loi quand il y a un litige entre particuliers, et décide, en refusant l'application d'une loi incriminée, si selon son jugement elle est inconstitutionnelle, mais cette action n'a pas pour effet d'annuler la loi qui subsiste parce que la décision de la Cour n’a que des effets dans les limites du procès, si bien que, dans d'autres cas similaires, un nouveau jugement et une nouvelle sentence seront nécessaires. De cette manière la Cour Suprême exerce sa fonction judiciaire et non pas législative $\aleph^{33}$.

De 1903 à 1935, le rôle de la Cour Suprême évolua peu. Les recours pour inconstitutionnalité dans la jurisprudence bolivienne restèrent marginaux. En fait, il y eut de nombreuses difficultés dans la mise en place de ces recours dont la procédure était toujours mal connue des juges et avocats. L'exégèse des décisions de la Cour Suprême en matière de déclaration d'inconstitutionnalité fournit peu de précisions sur l'utilisation de ce type de recours dans la jurisprudence ${ }^{34}$. On peut cependant relever quelques thèmes abordés par la jurisprudence pendant cette période, même si celle-ci consacrait des décisions parfois opposées. Ainsi, dans un arrêt de 1905, un tribunal de première instance affirme être compétent pour déclarer indirectement la constitutionnalité des lois ${ }^{35}$. En effet, dans les cas où les tribunaux de première instance déclaraient une norme inconstitutionnelle, celle-ci ne pouvait s'appliquer dans l'affaire en $\operatorname{cours}^{36}$. Mais la Cour Suprême était alors automatiquement saisie pour statuer sur la constitutionnalité de la norme incriminée ${ }^{37}$. En

\footnotetext{
31 Jaime Urcullo Reyes, "El control constitucional en Bolivia antes de 1994”, op. cit., p. 34.

${ }^{32}$ L'auteur se référait dans ce discours à la Cour Suprême des États-Unis.

${ }^{33}$ Corte Suprema, Discursos de los presidentes de la Corte Suprema, op. cit., p. 445.

34 Pour cette interprétation de la jurisprudence en matière de contrôle de constitutionnalité, nous nous référons aux ouvrages d'Hernando Siles, Procedimiento Civil e Historia de la Jurisprudencia, La Paz, Bolivia, Edición, Don Bosco, 1916 ; Roberto Zapata, Estudio Crítico de la Jurisprudencia nacional, La Paz, Bolivia, Escuela Tipográgafica, 1928 ; Samuel Oropeza, Compilación de las leyes del Procedimiento Civil Boliviano, Sucre, Bolivia, Imprenta Boliviana, 1904, Tomo Segundo.

${ }_{35}$ Corte Suprema de Justicia, Gaceta Judicial Boliviana, La Paz, Bolivia, Ediciones de la Corte Suprema, Bolivia, 1905, N²10, p. 1137.

36 Ibidem, p. 1137.

${ }^{37}$ Ibidem, p. 1137.
} 
cas de décision qui déclarait l'inconstitutionnalité d'une norme, la Cour Suprême pouvait écarter son application dans le procès en cours sans pour autant l'annuler dans l'absolu ${ }^{38}$. Cet arrêt confirmait la saisine automatique de la Cour Suprême dans le cas où le juge de première instance se prononçait sur l'inconstitutionnalité d'une norme. Mais, dans un sens contraire, on trouvait un arrêt de la Cour Suprême de 1912 qui précisait que si la Cour de District, conformément à la loi de municipalité, a la faculté pour statuer sur les élections municipales, elle n'a pas de compétence pour résoudre les cas d'inconstitutionnalité des résolutions ${ }^{39}$.

Cet arrêt niait donc toute compétence aux juges de première instance et de second degré pour examiner, même à l'occasion d'un procès, un " recours direct en nullité » visant à examiner la constitutionnalité d'une norme. Il y eut ensuite quelques arrêts relatifs à la déclaration d'inconstitutionnalité de moindre importance. Un arrêt de la Cour Supérieure de District de 1914 déclara que l'ordonnance municipale qui fixait les mesures du pain et son prix contrairement à la règle économique fixée par les intéressés ne respectait pas la règle du libre commerce et devait être déclarée inconstitutionnelle ${ }^{40}$.

Dans ce cas, la Cour Suprême ne se prononça pas sur les conclusions de la Cour Suprême de District. Par ailleurs, dans un arrêt de 1924, la Cour Suprême se déclara incompétente pour contrôler à l'occasion d'un procès une norme qui abordait des thèmes politiques relevant du pouvoir exécutif ${ }^{41}$. Cependant, dans un autre arrêt de 1925, la Cour Suprême précisa que le contrôle de constitutionnalité pouvait se réaliser à l'occasion d'un litige entre un particulier et un fonctionnaire public chargé d'appliquer une norme inconstitutionnelle émanant du pouvoir exécutif ${ }^{42}$.

L'analyse de ces arrêts démontre à la fois la marginalité des recours intentés pour inconstitutionnalité dans le système judiciaire du pays et la difficulté pour les tribunaux d'établir une jurisprudence constante dans ce domaine lors de la première moitié du XX siècle. Il faut attendre la Constitution du 30 octobre de 1938 qui institua un nouveau recours, l'habeas corpus, pour voir légèrement augmenter le nombre des recours juridictionnels visant à examiner la constitutionnalité d'une norme ${ }^{43}$. Le recours pour habeas corpus établissait qu'une décision judiciaire pouvait faire l'objet d'un « recours direct en nullité » pour inconstitutionnalité devant la Cour Suprême de Justice, dans le cas où l'on constatait une atteinte aux droits fondamentaux d'une personne, reconnus dans la Constitution ${ }^{44}$.

Ce recours pouvait être intenté par la personne qui avait subi une atteinte à ses droits fondamentaux ou par un tiers qui l'aurait constatée ${ }^{45}$. En fait, toute personne qui estimait être poursuivie, détenue, attaquée en justice ou arrêtée, indûment ou illégalement, pouvait intenter une action en justice, devant la Cour Suprême, la Cour de District ou devant un juge de première instance. Le tribunal saisi devait rendre son jugement dans les vingt-quatre heures au cours d'une audience ordonnant, s'il y avait lieu, la mise en liberté du requérant et faisant en sorte que les irrégularités légales soient réparées ${ }^{46}$. La sentence était portée

\footnotetext{
${ }^{38}$ Corte Suprema de Justicia, Gaceta Judicial Boliviana, La Paz, Bolivia, 1912, N 446, p. 73.

${ }^{39}$ Ibidem, $\mathrm{N}^{\circ}$ 649, p. 9.

${ }^{40}$ Corte Superior de Distrito, Gaceta Judicial Boliviana, La Paz, Bolivia, 1914, N 397, p. 279.

${ }^{41}$ Corte Suprema de Justicia, Gaceta Judicial Boliviana, La Paz, Bolivia, 1924, N 994, p. 28.

${ }^{42}$ Corte Suprema de Justicia, Gaceta Judicial Boliviana, La Paz, Bolivia, 1925, N 757, p. 34.

43 “Artículo 8 de la Constitución Política de 1938”, Ramón Salinas Mariaca, Las constituciones de Bolivia, op. cit., p. 240.

44 “Artículo 8 de la Constitución Política de 1938”, ibidem, p. 240.

45 Ibidem, p. 240

${ }^{46}$ Ibidem, p. 240.
} 
d'office en révision devant la Cour Suprême de Justice qui examinait la constitutionnalité de la norme incriminée 47 . Les fonctionnaires de l'État ou les particuliers qui s'opposaient à l'exécution des décisions judiciaires devaient comparaittre devant le juge qui avait eu connaissance de l'habeas corpus, et pouvaient être inculpés d'«atteinte aux garanties constitutionnelles $»^{48}$.

À partir de la mise en place de l'habeas corpus, le contrôle de constitutionnalité commença à devenir plus fréquent ${ }^{49}$. Dans un arrêt de 1939, la Cour Suprême estima que le décret suprême du 28 juillet 1939, qui créait un tribunal de responsabilité pour juger les hauts fonctionnaires de l'État, détournait la loi de responsabilité du 31 octobre 1884 et que, dès lors, il était inconstitutionnel et ne pouvait être appliqué50.

Il s'agissait, en l'espèce, d'un décret suprême émis par le général Germán Busch en 1939, et destiné à créer un tribunal spécial pour juger les délits commis par des hauts fonctionnaires de l'État ${ }^{51}$. L'inconstitutionnalité du décret ne résidait pas dans la création d'un tribunal exceptionnel, mais dans l'édiction d'une mesure qui faisait déjà l'objet d'une réglementation par la Constitution. En effet, la Constitution de 1938 disposait dans son article 8 alinéa 2 que : Les fonctionnaires publics ou particuliers qui résistent aux décisions judiciaires, dans les cas prévus par cet article, seront inculpés d'atteinte aux garanties constitutionnelles, en tout temps. Ils (Les fonctionnaires publics ou particuliers) ne pourront se servir de l'excuse d'avoir accompli des ordres supérieurs ${ }^{52}$.

À partir de 1938, la doctrine juridique bolivienne constata donc une amélioration institutionnelle ${ }^{53}$. Mais la jurisprudence en matière de contrôle de constitutionnalité continua à être considérée comme une exception ${ }^{54}$. Ce n'est qu'avec la Constitution de 1967 que de nouveaux recours en matière de contrôle de constitutionnalité furent instaurés dans l'édifice juridictionnel bolivien.

Premièrement, la Constitution de 1967 institua le recours pour amparo constitutionnel55. Ce recours pouvait être intenté par un particulier lorsqu'il n'existait pas d'autres moyens juridiques pour la protection de ses droits. L'amparo constitutionnel pouvait s'exercer contre tout acte illégal ou omission illicite des fonctionnaires ou des

\footnotetext{
${ }^{47}$ Ibidem, p. 240.

48 Ibidem, p. 240

49 Sur ce sujet, voir Francisco Fajardo, "Inconstitucionalidad de Leyes y Decretos Supremos", Revista de Estudios Jurídicos, Políticos y Sociales, Sucre Bolivia, Publicación de la Facultad de Derecho, Julio 1947 ; Carlos Morales Guillén, Código de procedimiento Civil concordado y anotado, La Paz, Bolivia, Gisbert y Cía, Libreros Editores 1982, 2e Edición, p. 1237.

${ }^{50}$ Corte Suprema de Justicia, Gaceta Judicial Bolivina, La Paz, Bolivia, 1939, n¹192, p. 5.

${ }^{51}$ República de Bolivia, Redactor de la Convención Nacional del año 1938, La Paz, Bolivia, Edición República de Bolivia, Litografías e Imprentas Unidas, 1938, p. 80.

52 “Artículo 8 de la Constitución Política de 1938", Ramón Salinas Mariaca, Las Constituciones de Bolivia, op. cit., p. 240.

53 Carlos Arce y Jaime Urcullo, Diccionario de Jurisprudencia Boliviana, La Paz, Bolivia, Imprenta Don Bosco, 1981, Tomo I, pp. 298-299.

54 «La demande d'inconstitutionnalité n'a pas besoin d'un jugement préalable sur des faits concrets». Corte Suprema de Justicia, Gaceta Judicial Boliviana, La Paz, Bolivia 1941, n¹285, p. 6. "Le décret suprême du 12 juillet 1946 qui se limite à appliquer la loi du $1^{\text {er }}$ décembre 1944, se référant aux charges sur les boissons gazeuses, n'est pas inconstitutionnel ", Corte Suprema de Justicia, Gaceta Judicial Boliviana, La Paz, Bolivia, 1946, n¹305, p. 183. « On ne peut demander directement l'inconstitutionnalité d'un décret ou d'une loi, sans intenter préalablement l'action de pur droit contre la personne ou l'autorité qui tente de rendre effective la disposition d'inconstitutionnalité pour que la Cour Suprême puisse statuer dans le cas conforme à l'attribution constitutionnelle ». Corte Suprema de Justicia, Gaceta Judicial Bolivina, La Paz, Bolivia, 1948, n¹424, p. 47.

55 “Artículo 18 de la Constitución Política de 1967”, Ramón Salinas Mariaca, Las constituciones de Bolivia, op. cit., pp. 356-357.
} 
particuliers qui restreignaient, supprimaient ou menaçaient les droits et garanties de la personne reconnus par la Constitution et les lois. Le recours pour amparo constitutionnel pouvait être intenté par la personne qui s'estimait offensée ou par une autre agissant en son nom, devant les Cours Supérieures de District et devant les juges de première instance devant la Cour Suprême ${ }^{56}$. L'amparo constitutionnel institua donc une procédure élargie qui permettait d'invoquer le respect de la Constitution et donc des droits fondamentaux, pour tout acte émanant d'une institution publique, judiciaire, voire même d'un particulier.

Deuxièmement, la Constitution de 1967 instaura le «recours pour illégalité de l'imposition $\aleph^{57}$. Ce recours juridictionnel était essentiellement destiné à contrôler la légalité des impôts créés par les pouvoirs exécutif et législatif ${ }^{58}$. En fait, si la Constitution de 1967 reconnaissait la faculté de l'État à créer des impôts au moyen d'une loi votée par le Parlement, cette activité devait être conforme aux principes établis dans la Constitution ${ }^{59}$. Dans le cas où l'État ne respectait pas ces principes constitutionnels, toute personne s'estimant lésée pouvait saisir la Cour Suprême de Justice sur la base d'un « recours pour illégalité de l'imposition $»^{60}$. La Constitution de 1967 reprenait aussi tous les autres recours juridictionnels pour inconstitutionnalité institués par les Chartes précédentes et en ajoutait d'autres ${ }^{61}$.

Mais, malgré quelques cas de jurisprudence ${ }^{62}$, le contrôle de constitutionnalité demeura encore marginal dans le système judiciaire bolivien. Il y eut plusieurs raisons à cela.

Tout d'abord, dans la deuxième partie du XX $\mathrm{XX}^{\mathrm{e}}$ siècle, jusqu'en 1978, la Bolivie fut gouvernée, dans une grande partie de son histoire, par des dictateurs arrivés au pouvoir après un coup d'État ${ }^{63}$. Dès lors, dans ce contexte, l'utilisation des recours destinée à prouver l'inconstitutionnalité d'une norme émanant du pouvoir exécutif apparaissait inefficace. L'absence de recours juridictionnels pour contester la constitutionnalité d'une norme durant les périodes de dictature fut même interprétée par certains juristes boliviens comme la reconnaissance du caractère légal des décisions émises par les gouvernements de facto $^{64}$. En effet, une partie de la doctrine considérait qu'une norme émanant du pouvoir exécutif devait être considérée comme légale tant qu'elle n'avait pas été reconnue inconstitutionnelle par un juge et cela même si un dictateur était à l'origine de cette norme ${ }^{65}$. Dès lors, cette situation n'était pas de nature à favoriser les recours pour inconstitutionnalité à la Cour Suprême.

Ensuite, l'ensemble des contrôles institués au cours de l'histoire bolivienne et synthétisé par la Constitution de 1967 ne s'appliquait qu'entre les parties d'un procès. La norme déclarée inconstitutionnelle continuait à subsister. Ceci produisait dans la pratique une certaine insécurité dans la jurisprudence car le résultat d'une sentence n'avait pas

\footnotetext{
56 “Artículo 18 de la Constitución Política de 1967”, Ramón Salinas Mariaca, Las Constituciones de Bolivia, op. cit., p. 356.

57 “Artículo 26 de la Constitución Política de 1967”, ibidem, p. 356.

58 “Artículo 26 de la Constitución Política de 1967", ibidem, p. 356.

59 “Artículos 26, 27, 29 de la Constitución de 1967”, ibidem, pp. 358-359.

60 “Artículo 26 de la Constitución Política de 1967", ibidem, p. 356.

61 "Recursos directos de nulidad, article 122 de la Constitución Política de 1967”, Ramón Salinas Mariaca, Las constituciones de Bolivia, ibidem, p. 380. "Recursos de habeas corpus, artículo 18 de la Constitución Política de 1967", ibidem, p. 356.

62 Jaime Urcullo Reyes, "El control constitucional en Bolivia antes de 1994”, op. cit., pp. 39-68.

${ }^{63}$ Voir sur ce sujet Carlos D. Mesa Gisbert, Presidentes de Bolivia : entre urnas y fusiles (el poder ejecutivo : los ministros de Estado), La Paz, Bolivia, Editorial Gisbert y Cía. S.A., 1990.

${ }^{64}$ Francisco Fajardo, "Inconstitucionalidad de Leyes y Decretos Supremos", op. cit., p. 74.

${ }^{65}$ Ibidem, p. 74.
} 
d'effet sur un jugement ultérieur ${ }^{66}$. Dès lors, la jurisprudence de la Cour Suprême en matière de contrôle de constitutionnalité n'était pas constante et devenait parfois contradictoire. Sur cette situation Francisco Fajardo précisait: Qu'une décision rendue par la Cour Suprême pour inconstitutionnalité pouvait donner lieu à un résultat opposé dans une autre décision ${ }^{67}$.

\section{LA CONSÉCRATION DU CONTRÔLE DE CONSTITUTIONNALITÉ DANS LA CONSTITUTION BOLIVIENNE DE 2009}

Ces types de recours qui déclaraient l'inconstitutionnalité d'une norme seulement entre les parties d'un procès, doublés d'une certaine réserve des juges de la Cour Suprême, notamment dans les périodes de dictature, ne favorisèrent donc pas l'émergence d'un réel contrôle de constitutionnalité et d'une jurisprudence abondante et constante sur ces sujets. Ce n'est qu'à l'occasion de la réforme constitutionnelle de 1994 que l'on observa la consécration d'un véritable contrôle de constitutionnalité en Bolivie.

Ce fut la loi º1585 du 12 août 1994, réformant la Constitution de 1967, qui inscrivit le Tribunal Constitutionnel dans l'édifice juridictionnel bolivien. Selon l'article 116 de la Constitution de 1967 réformée en 1994 et 2004 : Le Pouvoir Judiciaire est exercé par la Cour Suprême de Justice de la Nation, le Tribunal Constitutionnel, les Cours Supérieures de District, les tribunaux et les juges d'instance, les autres tribunaux et les judicatures que la loi établitit ${ }^{68}$.

Le Tribunal Constitutionnel devenait ainsi un organe du pouvoir judiciaire destiné uniquement au contrôle de constitutionnalité ${ }^{9}$, la Cour Suprême perdant cette compétence $^{70}$. La loi $\mathrm{N}^{\circ} 1836$ du $1^{\text {er }}$ avril 1998 compléta par la suite les dispositions relatives

\footnotetext{
${ }^{66}$ Jorge Asbun, Análisis jurídico-politico de la reforma constitucional, Santa Cruz, Bolivia, Universidad Privada de Santa Cruz de la Sierra, Santa Cruz, 1994, pp. 28-29.

${ }^{67}$ Francisco Fajardo, "Inconstitucionalidad de Leyes y Decretos Supremos”, op. cit., p. 350.

68 "Artículo 116 de la Constitución de 1967 reformada el 12 de Agosto de 1994 y aprobada por ley N 1615 del 6 de Febrero de 1995”, op. cit., p. 18.

69 “Artículo 119 de la Constitución de 1967 reformada el 12 de Agosto de 1994 y aprobada por ley N 1615 del 6 de Febrero de 1995, ibidem, p. 19.

${ }^{70}$ L'article 116 de la Constitution de 1967 réformée en 1994 précisait : I - Le Pouvoir Judiciaire est exercé par la Cour Suprême de Justice de la Nation, le Tribunal Constitutionnel, les Cours Supérieures de District, les tribunaux et les juges d'instance, les autres tribunaux et les judicatures que la loi établit. La loi détermine l'organisation et les attributions des tribunaux et des judicatures de la République. Le Conseil de la Judicature fait partie du Pouvoir Judiciaire. II - Des tribunaux ou des judicatures d'exception ne peuvent être établis. III - La faculté de juger dans la voie ordinaire, contentieuse et contentioadministrative et celle de faire exécuter le jugement incombent à la Cour Suprême et aux juges respectifs sous le principe de l'unité juridictionnelle. IV - Le contrôle de constitutionnalité est exercé par le Tribunal Constitutionnel. $V$ - Le Conseil de la Judicature est l'organe administratif et disciplinaire du Pouvoir Judiciaire. VI - Les Magistrats et les Juges sont indépendants dans l'administration de la Justice et ne sont soumis qu'à la Constitution et la loi. Ils ne pourront être révoqués de leurs fonctions sans sentence exécutoire préalable. VII - La loi établit l'échelonnement Judiciaire et les conditions d'inamovibilité des Membres des Cours, des Magistrats, des Conseillers et des Juges. VIII - Le Pouvoir Judiciaire a une autonomie économique et administrative. Le Budget Général de la Nation lui attribuera un budget annuel fixe qui sera centralisé dans le Trésor Judiciaire qui dépend du Conseil de la Judicature. Le Pouvoir Judiciaire n'est pas autorisé à créer ou à établir des taxes ni des droits judiciaires. IX - L'exercice de la judicature est incompatible avec toute autre activité publique et privée rémunérée à l'exception de la chaire universitaire. X-La gratuité, la publicité, la célérité et la probité dans les procès sont des conditions essentielles de l'administration de la justice. Le Pouvoir Judiciaire est responsable de pourvoir à la défense légale gratuite des indigents, ainsi qu'aux services de traduction lorsque leur langue maternelle n'est pas l'espagnol. "Artículo 116 de la Constitución de 1967 reformada el 12 de Agosto de 1994 y aprobada por ley n 1615 del 6 de febrero de 1995”, ibidem, p. 19.
} 
à l'organisation du Tribunal Constitutionnel dans l'édifice du pouvoir judiciaire ${ }^{71}$. Selon les dispositions de la loi du $1^{\text {er }}$ avril 1998, le Tribunal Constitutionnel devenait gardien de la Constitution $^{72}$. Il était également chargé de veiller au respect de l'ordre démocratique, des droits fondamentaux et garanties constitutionnelles de la personne ${ }^{73}$.

Le Tribunal Constitutionnel se composait de cinq membres élus parmi des juges, des procureurs, professeurs d'université, professionnels disposant d'un titre d'avocat et ayant déjà dix ans d'exercice ${ }^{74}$. Les membres du Tribunal Constitutionnel étaient désignés par les deux tiers du Congrès pour un mandat de dix $a^{75} s^{75}$ La création du Tribunal Constitutionnel suscita à l'époque de nombreuses critiques dans la doctrine juridique bolivienne. Une partie de cette doctrine estimait que la création de ce Tribunal dépossédait la Cour Suprême de ses attributions naturelles ${ }^{76}$. Edgar Oblitas, ancien président de la Cour Suprême, précisait à ce titre que le Tribunal Constitutionnel situé au-dessus de la Cour Suprême constituait une véritable aberration ${ }^{77}$.

En effet, selon cet auteur, en perdant sa fonction principale du contrôle de constitutionnalité, la Cour Suprême cessait d'être un pouvoir d'État ${ }^{78}$. Les magistrats de la Cour Suprême écrivaient, dans un document daté du 29 mars 1993 à propos des débats de l'époque sur la réforme de la Constitution de 1967, que le Tribunal Constitutionnel constituait une mesure attentatoire à l'indépendance du pouvoir judiciaire et que la constitutionnalisation du Tribunal Constitutionnel était un véritable coup d'État ${ }^{79}$.

Mais une autre partie de la doctrine juridique se prononça en faveur de la création d'un Tribunal Constitutionnel. Ainsi, Jaime Urcullo Reyes estimait que la création du Tribunal Constitutionnel ne retirait pas à la Cour Suprême ses attributions naturelles, mais transférait seulement ses fonctions relatives au contrôle de constitutionnalité ${ }^{80}$.

La Cour Suprême gardait ses attributions principales de Tribunal Suprême de Cassation en matière civile, pénale, commerciale, de travail, de famille, fiscale et minière ${ }^{81}$. Dans le même sens, Jorge Asbún estimait que l’incorporation du Tribunal Constitutionnel à l’ordre juridique bolivien apportait de nombreuses améliorations au système judiciaire ${ }^{82}$. En

\footnotetext{
${ }^{71}$ María Verónica Oblitas F., Ley del Tribunal Constitucional, Ley $N^{\circ} 1836$ del $1^{\circ}$ de abril de 1998, Comentada y Concordada, La Paz, Cochabamba, Bolivia, Editorial Los Amigos del Libro, 1998, p. 57.

${ }^{72}$ Fernando Oblitas Mendoza, Notas sobre reformas judiciales y constitucionales, La Paz, Bolivia, Ediciones producciones cima, 1997.

73 República de Bolivia, “Artículo 1 de la ley n 1836, Ley del $1^{\circ}$ de abril 1998”, María Verónica Oblitas F., Ley del Tribunal Constitucional, Ley $n^{\circ} 1836$ del $1^{\circ}$ de Abril de 1998, Comentada y Concordada, La Paz, Cochabamba, Bolivia, Editorial los Amigos del Libro, 1998, p. 37.

74 Pour être élu magistrat du Tribunal Constitutionnel, il est nécessaire de réunir les mêmes conditions que pour être élu magistrat de la Cour Suprême de Justice. Ces conditions étaient définies dans les articles 61 et 64 de la Constitution de 1967 réformée en 1994. "Artículo 117 de la Constitución de 1967 reformada el 12 de Agosto de 1994 y aprobada por ley N¹615 del 6 de Febrero de 1995”, op. cit., p. 19.

75 “Artículo 119 de la Constitución de 1967 reformada el 12 de Agosto de 1994 y aprobada por ley n 1615 del 6 de febrero de 1995", op. cit., p. 19.

76 Gabriel Peláez Gantier, "El Tribunal Constitucional y la Corte Suprema”, La Paz, Bolivia, Presencia, Sección "Efeméride", 25 de Mayo de 1993.

77 Edgar Oblitas, "Discursos e informe de la gestión de 1993”, Sucre, Bolivia, 3 de Enero de 1994, pp. 29-30.

${ }^{78}$ Samuel B. Abad Yupanqui, "La reforma constitucional en Bolivia", Boletín de la Comisión Andina de Juristas, Lima, Septiembre, 1994, n 42, pp. 9-18.

${ }^{79}$ Marcel Galindo Ugarte, ¿ Tribunal Constitucional o Corte Suprema? una contribución al debate, La Paz, Bolivia, Fundación Milenio, 1994, p. 11.

${ }^{80}$ Jaime Urcullo Reyes, "El control constitucional en Bolivia antes de 1994”, op. cit., p. 59.

${ }^{81}$ Fernando Aguirre, "Las reformas en el poder judicial", Congreso del H. Senado Nacional, Reflexiones sobre la ley de necesidad de reforma de la Constitución Política del Estado, La Paz, Bolivia, Fundación Milenio, ILDIS, Ediciones del Senado Nacional, 1994, p. 99-100.

${ }^{82}$ Jorge Asbún, "Análisis jurídico-político de la reforma constitucional”, op. cit., p. 27.
} 
effet, le citoyen voyait ainsi ses droits fondamentaux protégés par un organe spécialisé 83. Par ailleurs, selon cet auteur, le Tribunal Constitutionnel permettait de pallier les problèmes du retard de la justice et du manque de formation des juges de première instance en matière du contrôle de constitutionnalitée ${ }^{84}$.

Le Tribunal Constitutionnel devint donc, avec la réforme constitutionnelle de 1994, l'organe juridictionnel destiné au contrôle de la constitutionnalité des normes. Ce contrôle portait sur plusieurs points. Le Tribunal Constitutionnel était chargé du contrôle de constitutionnalité des dispositions légales dans leur ensemble85. La loi du $1^{\text {er }}$ avril 1998 disposait ainsi que: La Constitution était violée lorsqu'un texte de loi, un décret, une résolution ou un acte émanant d'une autorité publique ou d'un particulier avait, dans ses effets ou son interprétation dans un cas concret, des dispositions contraires à la Constitution $^{86}$.

Ce processus fut nettement amplifié par la Constitution de 2009. Cette Constitution, caractérisée par une invention constitutionnelle spécifique en changeant l'ordonnancement juridique du pays, notamment par la création de juridictions réservées aux communautés indigènes, ne modifiait cependant pas les compétences du Tribunal Constitutionnel. L'article 196 de la Constitution de 2009 précisait :

« I Le Tribunal Constitutionnel Plurinational veille à la suprématie de la Constitution, exerce le contrôle de constitutionnalité et protège le respect et la vigueur des droits et des garanties constitutionnels.

II Dans sa fonction interprétative, le Tribunal Constitutionnel Plurinational appliquera, comme critère d'interprétation, de préférence la volonté du Constituant en accord avec ses documents, actes et résolutions ainsi que la teneur littérale du texte $»^{87}$.

De plus, les magistrats du Tribunal Constitutionnel Plurinational, à l'instar des autres magistrats des Tribunaux, étaient élus directement par le peuple ${ }^{88}$ mais devaient obligatoirement inclure une représentation du système indigène originaire paysan ${ }^{89}$. Les compétences du Tribunal Constitutionnel étaient définies par l'article 202 de la Constitution de 2009 et, dans l'ensemble, ne changeaient pas de celles inscrites dans la Constitution de 1967 réformée en 1994 et 2004. Le Tribunal Constitutionnel Plurinational était juge de dernière instance pour les sujets de pur droit sur l'inconstitutionnalité des lois, les statuts des autonomies, les Chartes organiques, les décrets et tout autre type d'ordonnances et de résolutions non judiciaires ${ }^{90}$.

\footnotetext{
${ }^{83}$ Ibidem, p. 27.

84 Gualberto Dávalos, "La posición de la Corte Suprema de Justicia”, Congreso del H. Senado Nacional, Reflexiones sobre la ley de necesidad de reforma de la Constitución Política del Estado, La Paz, Bolivia, Fundación Milenio, ILDIS, Ediciones del Senado Nacional, 1994, p. 132.

85 On entend par «dispositions légales", les lois, décrets ou autres résolutions du corps administratif. República de Bolivia, "Artículo 7 inc. 1 et 2 de la ley N¹836, Ley del $1^{\circ}$ de Abril 1998”, María Verónica Oblitas F., Ley del Tribunal Constitucional, Ley No1836 del $1^{\circ}$ de Abril de 1998, Comentada y Concordada, Op. cit., p. 39.

86 “Artículo 3 de la ley N¹836, Ley del $1^{\circ}$ de Abril 1998", ibidem, p. 38.

87 “Artículo 196 de la Constitución Política del Estado promulgada el 7 de febrero de 2009”, República de Bolivia, Constitución Política del Estado promulgada el 7 de febrero de 2009, op. cit., p. 78.

88 “Artículo 198 de la Constitución Política del Estado promulgada el 7 de febrero de 2009”, ibidem, p. 79.

89 "Artículo 197 de la Constitución Política del Estado promulgada el 7 de febrero de 2009", ibidem, pp. 78-79.

${ }^{90}$ L'article 202 précisait : Sont des attributions du Tribunal Constitutionnel Plurinational en plus de celles établies par la Constitution et la loi, de connaître et résoudre:

1. En unique instance les sujets de pur droit portant sur l'inconstitutionnalité des lois, les statuts des autonomies, les Chartes organiques, les décrets et tout autre type d'ordonnances et de résolutions non judiciaires. Si l'action est de caratère abstrait, elle pourra être interposée par la présidente ou le président de la République, les sénateurs, les députés, les législateurs et les plus hautes autorités exécutives des entités territoriales autonomes.
} 
Mais la Constitution de 2009 accroissait les prérogatives du Tribunal Constitutionnel Plurinational afin d'harmoniser les décisions juridiques prises par la multitude de juridictions instaurées par le texte constitutionnel.

Tout d'abord, le Tribunal Constitutionnel Plurinational devait trancher les conflits de compétences entre le gouvernement plurinational et les entités territoriales autonomes et décentralisées ainsi que des conflits qui pourraient surgir entre ces entités ${ }^{91}$.

Ensuite, le Tribunal Constitutionnel Plurinational était compétent pour vérifier la valeur juridique des autorités indigènes originaires paysannes appliquée à un cas concret ${ }^{92}$.

Enfin, le Tribunal Constitutionnel Plurinational pouvait aussi trancher les conflits de compétences entre les juridictions indigènes originaires paysannes et la juridiction " agroenvironnementale $»^{93}$.

En fait, le Tribunal Constitutionnel Plurinational permit véritablement l'émergence d'un contrôle de constitutionnalité efficace, dans la mesure où toute norme émanant d'une autorité publique pouvait faire l'objet d'un recours pour inconstitutionnalité. Le Tribunal Constitutionnel Plurinational offrait donc véritablement aux acteurs sociaux des moyens juridiques substantiels et procéduraux de développer des stratégies juridiques propres ${ }^{94}$.

Pour la première fois, l'ensemble de la classe politique représentée au gouvernement fut d'accord sous deux législatures, de 1993 à 1997 sous la présidence de Gonzalo Sánchez de Lozada et de 1997 à 2002 sous la présidence de Hugo Banzer et de Jorge Quiroga, pour créer de nouvelles institutions structurant le système juridique bolivien. Mais, de surcroît, la Constitution de 2009 du gouvernement d'Evo Morales approuvée par un référendum par le peuple bolivien confirmait l'institutionnalisation du contrôle de constitutionnalité.

2. Les conflits de compétences et d'attributions entre les organes du pouvoir public.

3. Les conflits de compétences entre le gouvernement plurinational, les entités territoriales autonomes et décentralisées et les conflits entre elles.

4. Les recours contre les tributs, impôts, taxes, patentes, droits ou contributions créés, modifiés ou supprimés en contravention à ce qui est disposé dans cette Constitution.

5. Les recours contre les résolutions de l'Organe Législatif, quand ces résolutions affectent un ou plusieurs droits quelles que soient les personnes affectées.

6. La révision des actions de liberté, d'Amparo Constitutionnel, de protection, de privacité, populaire et d'accomplissement. Cette révision n'empêchera pas l'application immédiate et obligatoire de la résolution que résolvent les actions.

7. Les consultations de la présidente ou du président de la République, de l'Assemblée Législative Plurinationale, du Tribunal Suprême de Justice ou du Tribunal agro-environnemental sur la constitutionnalité des projets de loi. La décision du Tribunal Constitutionnel est d'accomplissement obligatoire.

8. Les consultations des autorités indigènes originaires paysannes sur l'application de leurs normes juridiques appliquées à un cas concret. La décision du Tribunal Constitutionnel Plurinational est obligatoire.

9. Le Contrôle préalable de constitutionnalité avant la ratification des traités internationaux.

10. La Constitutionnalité de la procédure de réforme partielle de la Constitution.

11. Les conflits de compétences entre la juridiction indigène originaire paysanne et la juridiction agro-environnementale.

12. Les recours directs de nullité. "Artículo 202 de la Constitución Política del Estado promulgada el 7 de febrero de 2009”, República de Bolivia, Constitución Política del Estado promulgada el 7 de febrero de 2009, op. cit., pp. 79-80.

91 "Artículo 202 inc 3 de la Constitución Política del Estado promulgada el 7 de febrero de 2009", ibidem, p. 80 .

92 “Artículo 202 inc 8 de la Constitución Política del Estado promulgada el 7 de febrero de 2009”, ibidem, p. 80 .

93 “Artículo 202 inc 11 de la Constitución Política del Estado promulgada el 7 de febrero de 2009”, ibidem, p. 80 .

94 Jean-Michel Blanquer, Entre "État de droit" et "Société de Droits" : l'Amérique latine à la recherche d'un concept directeur, Paris, Conférence prononcée pour l'inauguration de la BDIC, 1999, p. 111. 